\title{
Optical Coherence Tomography Angiography Findings in Polycystic Ovary Syndrome
}

\author{
Neslihan Parmak Yener ${ }^{1}$, Gulten Ozgen ${ }^{2}$, Ayse Tufekci ${ }^{1}$, Levent Ozgen ${ }^{3}$ and Gultekin Adanas Aydin ${ }^{2}$ \\ ${ }^{1}$ Department of Ophthalmology, Bursa Yuksek Ihtisas Education and Research Hospital, Bursa, Turkey \\ ${ }^{2}$ Department of Obstetrics and Gynaecology, Bursa Yuksek Ihtisas Education and Research Hospital, Bursa, Turkey \\ ${ }^{3}$ Department of Obstetrics and Gynaecology, Bursa City Hospital, Bursa, Turkey
}

\begin{abstract}
Objective: To evaluate microstructure and vascularity of macula and optic disc in polycystic ovary syndrome (PCOS) by optical coherence tomography (OCT) and OCT angiography (OCTA).

Study Design: Descriptive study.

Place and Duration of Study: Department of Ophthalmology and Department of Obstetrics and Gynecology, University of Health Sciences, Yüksek Ihtisas Training and Research Hospital, Bursa, Turkey between January and June 2019.

Methodology: Fifty-nine patients with PCOS and 35 healthy controls were enrolled in the study. Foveal avascular zone (FAZ), the capillary density assessed in the superficial capillary plexus (SCP) and deep capillary plexus (DCP) in the macula and was also measured in optic disc using the OCTA. The thicknesses of the retinal nerve fiber (RNFL), ganglion cell complex (GCC), and macula were measured by OCT.

Results: The capillary density in the macula and optic disc region were not a significant difference between the PCOS patients and healthy controls.The parafoveal superior, inferior, and temporal quadrant thickness was significantly higher in the study group compared to control group ( $p=0.047, p=0.033$, and $p=0.033$, respectively). In patients with PCOS, there were negative correlations between IR and inferior RNFL, total and superior GCC thickness $(r=-0.29 p=0.027, r=-0.27 p=0.050, r=-0.31$ $p=0.029$, respectively).

Conclusion: Although no microvascular macular abnormalities were seen in PCOS patients, the parafoveal thickness significantly increased in all quadrants, except the nasal quadrant. In patients with PCOS, IR, dyslipidemia may affect the structural integrity of the retina. Further longitudinal follow-up studies are needed to determine whether PCOS has any effect on OCTA findings.
\end{abstract}

Key Words: Polycystic ovary syndrome, Retina, Optical coherence tomography, Optical coherence tomography angiography.

How to cite this article: Yener NP, Ozgen G, Tufekci A, Ozgen L, Aydin GA. Optical Coherence Tomography Angiography Findings in Polycystic Ovary Syndrome. J Coll Physicians Surg Pak 2021; 31(09):1057-1063.

\section{INTRODUCTION}

Polycystic ovary syndrome (PCOS) is one of the most prevalent endocrin disorders in women of reproductive age. The mean prevalence is reported as $4-18 \%$. ${ }^{1}$ The clinical manifestations include irregular menstrual cycles, chronic anovulation, and hyperandrogenism such as acne, hirsutism, and androgenic alopecia with characteristic image of polycystic ovaries on ultrasonography. ${ }^{2}$ Polycystic ovary syndrome presenting with insulin resistance (IR) and hyperandrogenism may cause metabolic syndrome, which is a multifactorial condition of obesity, hyperglycemia, dyslipidemia, diabetes mellitus (DM), and cardiovascular diseases. ${ }^{2}$

Correspondence to: Dr. Gulten Ozgen, Department of Obstetrics and Gynaecology, Bursa Yuksek Ihtisas Education and Research Hospital, Bursa, Turkey

E-mail:drgaslanozgen@yahoo.com.tr

Received: June 20, 2021; Revised: July 28, 2021;

Accepted: August 28, 2021

DOI: https://doi.org/10.29271/jcpsp.2021.09.1057
Sex hormones have receptors in anterior segment structures such as the conjunctiva, cornea, lens and also in the retina. It has been demonstrated that sex hormones affect the blood flow through the retinal receptors and show retinal neuroprotection. Estrogen induces vascular relaxation while progesterone and testosterone produce vasoconstriction. ${ }^{3}$

The previous optical coherence tomography (OCT) studies have shown certain discrepancies in the peripapillary retinal nerve fiber layer ( $p R N F L)$, macular thicknesses between the patients with PCOS, and healthy controls. ${ }^{4,5}$ In a study using color Doppler USG, the ocular blood flow was found significantly higher in the PCOS women than healty womens. ${ }^{6}$.n recent years, OCT angiography (OCTA) has been introduced to enable non-invasive visualisation of the blood flow. The OCTA is a new imaging method based on the split-spectrum amplitude-decorrelation angiography (SSADA) algorithm to provide quantitative analysis of the microvascular structures of the peripapillary region and macula by detecting motions of erythrocytes and visualising blood flow using serial OCT scans. ${ }^{\top}$ 
Retinopathy is a preclinical marker for many systemic diseases. Hormonal and metabolic disorders may induce retinal alterations in PCOS. However, to the best of authors' knowledge there is no study in the literature investigating ocular perfusion in PCOS patients by OCTA.

The aim of the present study was to investigate morphovascular alterations in the retina in patients with PCOS using a new, non-invasive retinal imaging method, OCTA; and identify the possible relationship with biochemical and hormonal parameters, thus understanding the preclinical effect of PCOS.

\section{METHODOLOGY}

This study was carried out among 59 patients with PCOS, and 35 healthy women. This descriptive study was conducted at the Department of Ophthalmology and Department of Obstetrics and Gynecology, University of Health Sciences, Yüksek Ihtisas Training and Research Hospital, Bursa, Turkey betweenJanuary and June 2019. The study protocol was approved by the local Ethics Committee (No. 2019 /01-39; dated: 02/01/2019). A written informed consent was obtained from all participants before any examination. The study was conducted in accordance with the principles of the Declaration of Helsinki. The participants with patients group underwent a detailed gynecological examination and transvaginal ultrasonography. In accordance with the 2003 Rotterdam criteria, the diagnosis of PCOS was made based on the presence of at least two of the following three feature; the exclusion of related disorders (i.e., Cushing syndrome, androgen-secreting tumors): i) oligo/anovulation, ii) clinical and/or biochemical hyperandrogenism, and iii) polycystic ovaries on ultrasound. For both groups, exclusion criteria were as follows: having a previous ocular surgery or trauma, glaucoma, conditions affecting the optic nerve or retina, high refractive errors; having a smoking history; having systemic diseases such as systemic hypertension, coronary artery disease, DM, lipid metabolism disorder, cerebrovascular disease, cardiovascular event, or obesity; having hormonal therapy or systemic agents, antihypertensives, antihyperlipidemics, corticosteroids, or antidepressants.

Data including age, weight, height, body mass Index (BMI), waist and hip circumferences, time to diagnosis of PCOS, medicines used, history of ocular and systemic diseases, and hormonal and laboratory test results were recorded. Insulin resistance (IR) was calculated using the homeostatic model assessment insulin resistance (HOMA-IR) formula [fasting glucose $(\mathrm{pUl} / \mathrm{L}) \times$ fasting insulin $(\mathrm{mmol} / \mathrm{L}) / 22.5]$. The patients with a HOMA score of $\geq 2.5$ were considered to have IR. The modified Ferriman-Gallwey (mFG) score was used to identify the degree of hirsutism. Blood samples were taken after a 12hour overnight fasting on days 3 to 5 of the menstrual cycle. Biochemical and hormonal tests including serum glucose, insulin, estradiol $\left(\mathrm{E}_{2}\right)$, 17-hydroxyprogesterone (17-OHP), total testosterone (TT), dehydroepiandrosterone sulfate (DHEA-S), follicle-stimulating hormone (FSH), luteinizing hormone (LH), total cholesterol (TC), low-density lipoprotein cholesterol (LDLC), high-densitylipoprotein cholesterol (HDL-C), triglyceride
(TG), thyroid-stimulating hormone (TSH), and prolactin levels were performed.

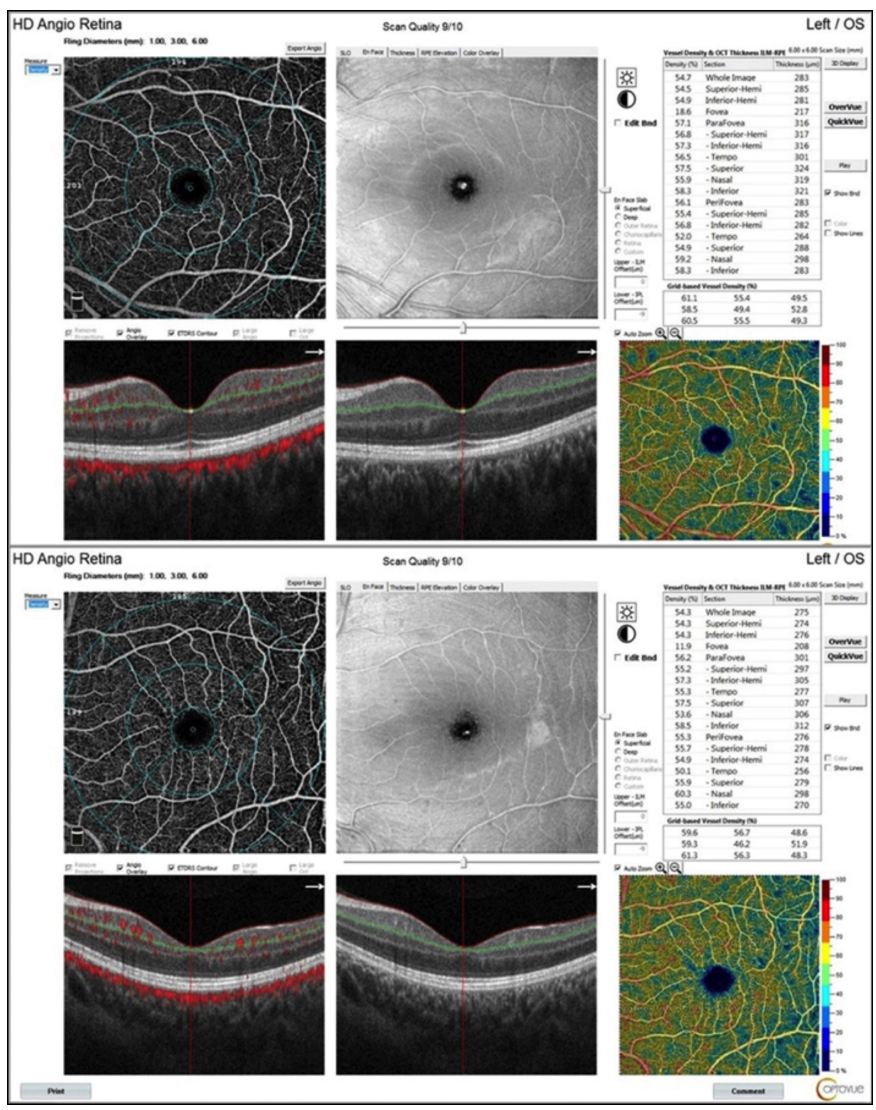

Figure 1: Impression of the deep capillary plexsus OCT angiogram of the macula in a patient with polycystic ovary syndrome (PCOS) (upper) and in a healthy control (below).

All study participants underwent a complete ophthalmologic examination including the best corrected visual acuity, intraocular pressure (Goldmann applanation tonometry), biomicroscopic and fundus examination. OCTA was performed by the AngioVue Imaging System (RTVue XR Avanti; Optovue Inc., Fremont, CA, USA) using the SSADA algorithm. It utilises a light source centred at $840 \mathrm{~nm}$ wavelength and $45 \mathrm{~nm}$ band width with a scan rate of 70,000 per second. ${ }^{10}$ OCTA imaging of the macula was obtained using a $6 \times 6-\mathrm{mm}$ scan. The superficial capillary plexus (SCP) and deep capillary plexus (DCP) density was measured in fovea, parafovea and perifovea according to ETDRS zones (temporal, superior, inferior, nasal) using the algorithm in computer software (Figure 1). Also FAZ area was automatically measured. Using the $4.5 \times 4.5 \mathrm{~mm}$ images, the vessel density of the optic disc region was measured (Figure 2).

The thicknesses of the PRNFL and GCC, macula thickness and volume were analyzed using a spectral domain OCT device (Avanti RTVue-XR 100, Optovue Inc., Fremont, CA, USA). Peripapillary RNFL thickness was measured with a $3.45 \mathrm{~mm}$ diameter ring section. GCC thickness including inner plexiform layer + ganglion cell layer + nerve fiber layer was obtained by GCC scanning protocol. The thickness map of the macula defined by ETDRS as fovea area, parafoveal area and perifoveal area were analyzed same device (Figure 3). The pupil was dilated with 
tropicamide \%1 eye drop. All OCT and OCTA scans were obtained from a single operator and same hour interval (10:00-13:00).Each eye was scanned three times. Low-quality images (defined as a SSI of $<60$ or a scan quality of $<6$ ) were excluded. The best quality image parameters were used for statistical analysis.

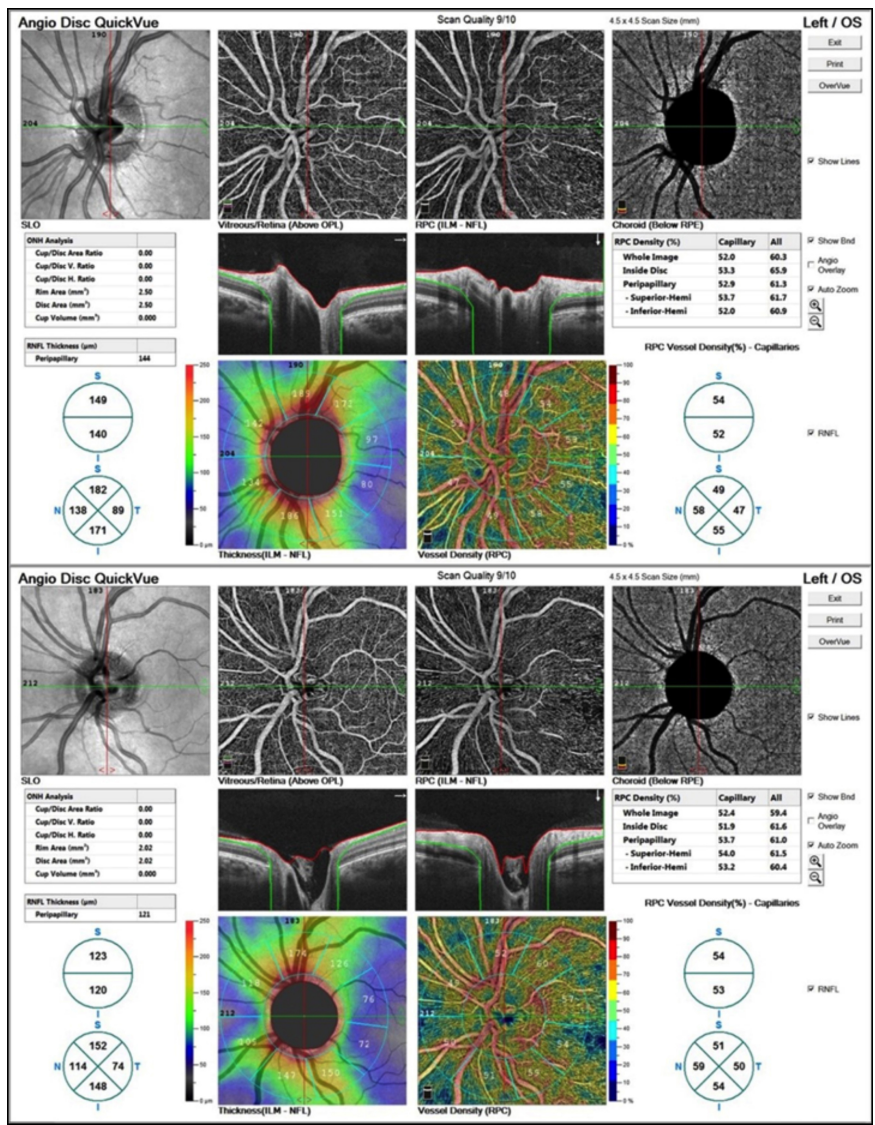

Figure 2: Impression of the capillary plexsus OCT angiogram of optic disc in a patient with polycystic ovary syndrome (PCOS) (upper) and in a healthy control (below).

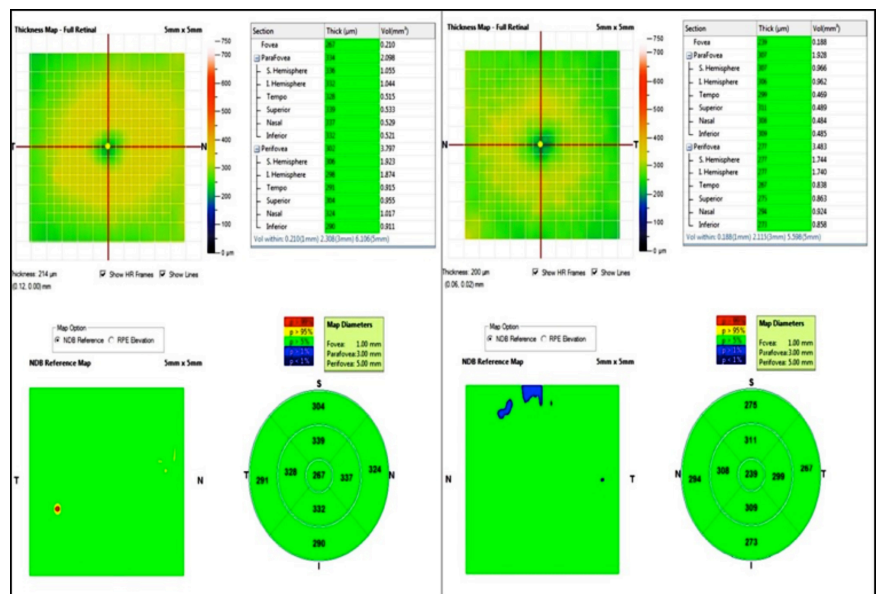

Figure 3: Example of OCT image of the macular thickness map in 9 zones according to ETDRS in a patient with polycystic ovary syndrome (PCOS) (right) and in a healthy control (left).

Statistical analysis was performed using the SPSS Version 23.0. Descriptive data were expressed in mean standard deviation (SD), median (min-max), ornumberand frequency. Kolmogorov-S- mirnov test was used to assess whether the variables followed a normal distribution. According to the normality test result, independent samples t-test and Mann-Whitney U-test were used to perform between-group comparisons. The Spearman correlation analysis was performed to evaluate possible correlations among the variables. A p-value less than 0.05 was considered statistically significant.

\section{RESULTS}

A total of 59 eyes of 59 PCOS patients, and 35 of 35 healthy controls were included.The median follow-up was two months. The median age was 22 years ranging from 17 to 37 in the PCOS group and 23 years ranging from 18 to 40 in the control group, indicating no statistically significant difference $(p=0.496)$. The body weight and $\mathrm{BMI}$ values were also similar between the groups $(p=0.338$ and $p=0.133)$. The intraocular pressure was within normal range in both groups. However, the mean $\mathrm{mFG}$ scores $(13.20 \pm 2.66)$ were statistically significantly higher in the PCOS group $(15.41 \pm 4.77)$ than the control group $(p=0.006)$. Demographic and clinical characteristics and anthropometric measurements of both groups are shown in Tablel-a.

Table I-a: Demographic and clinical characteristics and anthropometric measurements of PCOS and control groups.

\begin{tabular}{|l|l|l|l|}
\hline Variable & $\begin{array}{l}\text { PCOS } \\
(\mathbf{n = 5 9 )}\end{array}$ & $\begin{array}{l}\text { Control } \\
(\mathbf{n = 3 5 )}\end{array}$ & $\boldsymbol{p}$-value \\
\hline Age (years) & $22(17: 37)$ & $23(18: 40)$ & $0.496^{\mathrm{a}}$ \\
\hline $\begin{array}{l}\text { Intraocular pressure } \\
(\mathrm{mmHg})\end{array}$ & $18(14: 22)$ & $17(15: 21)$ & $0.058^{\mathrm{a}}$ \\
\hline Height (cm) & $162.73 \pm 5.68$ & $163.64 \pm 4.33$ & $0.428^{\mathrm{b}}$ \\
\hline Weight (kg) & $64(43: 125)$ & $61(48: 72)$ & $0.338^{\mathrm{a}}$ \\
\hline $\begin{array}{l}\text { Body mass index } \\
\left(\mathrm{kg} / \mathrm{m}^{2}\right)\end{array}$ & $23.56(15.77: 41.77)$ & $22.77(18.52: 26.23)$ & $0.133^{\mathrm{a}}$ \\
\hline $\begin{array}{l}\text { Waist circumference } \\
(\mathrm{cm})\end{array}$ & $67(58: 105)$ & $66(58: 76)$ & $0.163^{\mathrm{a}}$ \\
\hline $\begin{array}{l}\text { Hip circumference }(\mathrm{cm}) \\
\text { mFG score }\end{array}$ & $\begin{array}{l}97.58 \pm 10.69 \\
15.41 \pm 4.77\end{array}$ & $\begin{array}{l}95.67 \pm 3.46 \\
13.20 \pm 2.66\end{array}$ & $\begin{array}{l}0.212^{\mathrm{b}} \\
0.006^{\mathrm{b}}\end{array}$ \\
\hline $\begin{array}{l}\text { Data are given in median (min:max) and mean } \pm \text { standard deviation. a: } \text { Mann- } \\
\text { Whitney U-test; } \text { : Independent samples t-test; PCOS: Polycystic ovary syndrome; } \\
\text { mFG: Modified Ferriman-Gallwey. }\end{array}$ \\
\hline
\end{tabular}

Table I-b: RNFL, GCC, macular thickness, for PCOS patients and healthy controls.

\begin{tabular}{|c|c|c|c|}
\hline Variable & $\begin{array}{l}\text { PCOS } \\
(n=59)\end{array}$ & $\begin{array}{l}\text { Control } \\
(n=35)\end{array}$ & p-value \\
\hline \multicolumn{4}{|l|}{ RNFL thickness $(\mu \mathrm{m})$} \\
\hline Superior & $129(78: 178)$ & $132(111: 161)$ & $0.544^{\mathrm{a}}$ \\
\hline Temporal & $75.11 \pm 9.69$ & $73.06 \pm 7.16$ & $0.288^{b}$ \\
\hline Inferior & $141.53 \pm 21.29$ & $145.06 \pm 14.73$ & $0.391^{b}$ \\
\hline Nasal & $102.21 \pm 18.53$ & $102.97 \pm 15.49$ & $0.839^{b}$ \\
\hline Average & $112.83 \pm 13.06$ & $114.66 \pm 14.09$ & $0.527^{b}$ \\
\hline \multicolumn{4}{|l|}{ GCC thickness $(\mu \mathrm{m})$} \\
\hline Superior & $98.75 \pm 6.89$ & $97.63 \pm 7.11$ & $0.490^{b}$ \\
\hline Inferior & $101.06 \pm 6.39$ & $98.29 \pm 7.03$ & $0.076^{b}$ \\
\hline Total & $100.08 \pm 6.27$ & $97.97 \pm 6.74$ & $0.162^{b}$ \\
\hline \multicolumn{4}{|l|}{ Macular thickness $(\mu \mathrm{m})$} \\
\hline Superior parafoveal & $325(292: 357)$ & $316.50(213: 367)$ & $0.047^{a}$ \\
\hline Temporal parafoveal & $311(277: 340)$ & $302(234: 345)$ & $0.033^{a}$ \\
\hline Inferior parafoveal & $323(283: 363)$ & $314(215: 360)$ & $0.033^{a}$ \\
\hline Nasal parafoveal & $323(290: 361)$ & $316.50(214: 359)$ & $0.122^{a}$ \\
\hline Superior perifoveal & $292.73 \pm 19.24$ & $290.23 \pm 15.64$ & $0.583^{b}$ \\
\hline Temporal perifoveal & $275(233: 336)$ & $273.50(247: 309)$ & $0.952^{\mathrm{a}}$ \\
\hline Inferior perifoveal & $282.50(246: 341)$ & $281(257: 314)$ & $0.762^{a}$ \\
\hline Nasal perifoveal & $307(278: 345)$ & $301.50(279: 347)$ & $0.340^{\mathrm{a}}$ \\
\hline Fovea & $240.24 \pm 19.09$ & $241.82 \pm 16.75$ & $0.688^{b}$ \\
\hline \multicolumn{4}{|c|}{$\begin{array}{l}\text { Data are given in median (min:max) and mean } \pm \text { standard deviation. a: Mann- } \\
\text { Whitney U-test; b: Independent samples t-test; PCOS: Polycystic ovary } \\
\text { syndrome; RNFL: Retinal nerve fiber layer; GCC: Ganglion cell complex. }\end{array}$} \\
\hline
\end{tabular}


Table I-c. Quantitative OCTAanalysis results.

\begin{tabular}{|c|c|c|c|}
\hline Variables & $\begin{array}{l}\text { PCOS } \\
(n=59)\end{array}$ & $\begin{array}{l}\text { Control } \\
(n=35)\end{array}$ & $p$-value \\
\hline \multicolumn{4}{|c|}{ Superficial vessel density, \% } \\
\hline Whole-en-face & $48.80 \pm 2.82$ & $49.11 \pm 2.49$ & $0.596^{\mathrm{b}}$ \\
\hline Fovea & $17.74 \pm 6.40$ & $17.67 \pm 5.02$ & $0.959^{b}$ \\
\hline Parafovea & $51.77 \pm 2.99$ & $51.76 \pm 2.75$ & $0.994^{\mathrm{b}}$ \\
\hline Perifovea & $52.30 \pm 2.45$ & $51.94 \pm 4.75$ & $0.360^{\mathrm{b}}$ \\
\hline \multicolumn{4}{|l|}{ Deep vessel density, \% } \\
\hline Whole-en-face & $53.73 \pm 4.04$ & $54.47 \pm 3.53$ & $0.387^{\mathrm{b}}$ \\
\hline Fovea & $33.40 \pm 8.72$ & $35.10 \pm 5.17$ & $0.259^{\mathrm{b}}$ \\
\hline Parafovea & $56.70(48.60: 63.50)$ & $57.70(50.70: 61.90)$ & $0.283^{\mathrm{a}}$ \\
\hline Perifovea & $58.08(51.60: 63.70)$ & $58.40(50.70: 63.80)$ & $0.263^{\mathrm{a}}$ \\
\hline \multicolumn{4}{|c|}{ Optic disc-associated capillary density, \% } \\
\hline Whole-en-face & $50.28 \pm 2.42$ & $51.06 \pm 2.35$ & $0.402^{\mathrm{b}}$ \\
\hline Inside disc & $51.65 \pm 3.57$ & $50.52 \pm 4.90$ & $0.139^{b}$ \\
\hline Peripapillary & $\begin{array}{l}52.70 \\
(43.30: 63.90)\end{array}$ & $\begin{array}{l}53.90 \\
(46.70: 58.20)\end{array}$ & $0.137^{\mathrm{a}}$ \\
\hline $\begin{array}{l}\text { Foveal avascular zone, } \\
\mathrm{mm}^{2}\end{array}$ & $0.31 \pm 0.11$ & $0.29 \pm 0.08$ & $0.226^{\mathrm{b}}$ \\
\hline
\end{tabular}

Serum fasting blood glucose, fasting insulin, HOMA-IR, LH, TT, and TSH levels were significantly higher in the PCOS group $(p=0.026, p=0.036, p=0.035, p=0.019, p<0.001$ and $p=0.003$, respectively). The ratio participants with a HOMA-IR of $\geq 2.5$ was $28.8 \%(n=17)$ in the PCOS group versus $3 \%(n=1)$ in the control group, indicating a statistical significant difference $(p=0.003)$. There was no statistically significant difference in the thickness of pRNFL and GGC between the groups. The parafoveal superior, inferior, and temporal quadrant tkickness were statistically significantly higher in the PCOS patients compared to healthy controls $(p=0.047, p=0.033$, and $p=0.033$, respectively, Table I-b).

Table I-c shows the quantitative analysis results of macular and peripapillary OCTA scans. Although the mean FAZ was larger in the PCOS patients $\left(0.31 \pm 0.11 \mathrm{~mm}^{2}\right)$ than the control group $\left(0.29 \pm 0.08 \mathrm{~mm}^{2}\right)$, it did not show statistical significance $(p=0.226)$. There was no statistically significant difference in the macular SCP and DCP densities of whole-en-face, fovea, parafovea and perifovea between the PCOS patients and healthy controls. In addition, the capillary densities of the optic disc region in the whole image, the peripapillary and inside the optic disc, and the peripapillary region were similar $(p=0.137, p=0.139$, and $p=0.402$, respectively). Table II shows the correlation analysis results between PCOS biochemical and metabolic parameters and OCT, OCTA parameters.

\section{DISCUSSION}

Capillary densities in the macula and optic disc in this study were not different from healthy controls. There were some significant differences in macular OCT findings among the groups. It was found that parafoveal superior, inferior,and temporal quadrant thickness was significantly higher in the study group compared to control group.

In the literature, morphology of the retina in patient with PCOS have been previously investigated, but its microvascular structures have not been analysed. In the present study, the morphovascular alterations in the macula and optic disc of patients with PCOS using OCT and OCTA. PCOS-related metabolic and endocrine disorders may potentially affect the morphovascular parameters of the retina. Therefore, in the present study, the possible correlation of PCOS with biochemical and hormonal parameters was also investigated. In this study, the IR was increased in PCOS patients compared to healthy controls ( $28.8 \%$ vs. $3 \%$, respectively). In addition, the mean mFG scores, LH and TT levels, which are biochemical markers of hyperandrogenisim, were significantly higher in the PCOSpatients.

In a previous study using OCT in PCOS patients, Acmaz et al. reported an increased thickness of choroid and RNFL with thicker nasal and temporal perifovea. ${ }^{4}$ The authors concluded that nasal, temporal perifovea might be accepted as susceptible areas in PCOS patients.Similarly, Demir etal.found increased thicknesses of pRNFL at superior and inferior quadrant in PCOS patients than healthy women. ${ }^{5}$ In another study, de Souza-Junior et al. compared macular and pRNFL thickness between PCOS patients and healthy women and reported that PCOS might be a protective factor in the pRNFL, possibly through the action of testosterone, while it might be an aggressive factor in the macular region and reduce the $\mathrm{pRNFL}$ thickness when associated with metabolic abnormalities. ${ }^{8}$ In this study, similar thicknesses of RNFL and GCC was found between both groups. Increased LH and androgen levels have been shown to increase the nerve growth factor in PCOS. Testosterone has also been even to have neuronal and glial protective effects. ${ }^{9}$ In this study, although testosterone were higher in the PCOS patients than healthy controls, the RNFL and GCC thickness remained stable. This can be attributed to the fact that the coexistence of IR may mask the neuroprotective effects of hyperandrogenisim.

Insulin plays very important role associated with the cell metabolism, energy homeostasis, and neuronal functions. ${ }^{10}$ Despite established protective effects of insulin on retinal nerves, there is no study investigating retinal neurodegeneration in patients with IR. ${ }^{11}$ In previous studies including patients with diabetes and metabolic syndrome, the reduced RNFL thickness could be attributed to IR-relate neurodegeneration. ${ }^{12}$ Similarly, in this study, the presence of a negative relation of the inferior RNFL and total and superior GCC thickness with the HOMA-IR and insulin levels indicate that IR might have exerted a degenerative effect on retinal neurons. Furthermore, parafoveal region of the retina is a metabolically active territory, which contains an abundant amount of ganglion cells and macroglial cells consisting of Muller cells and astrocytes and providing neuroprotection metabolic support. ${ }^{13}$ Under normal circumstances, Muller cells and astrocytes are responsible for maintaining the structural integrity and cellular functions of the retina. ${ }^{14}$ In case of diabetic retinopathy, glial cells have been shown to be affected before the formation of neuronal cell degeneration, and activation of these cells release aberrant growth factors, reactive oxygen species, and inflammatory cytokines, resulting in retinal vascular dysfunction and neuronal degeneration. ${ }^{15}$ 


\begin{tabular}{|c|c|c|c|c|c|c|c|}
\hline OCT parameter & & Insulin & HOMA-IR & LDL-C & HDL-C & TC & TG \\
\hline \multicolumn{8}{|l|}{ Rnfl thickness $(\mu \mathrm{m})$} \\
\hline \multirow{2}{*}{ Inferior } & $r_{5}$ & -0.20 & -0.29 & -0.27 & -0.10 & 0.02 & 0.01 \\
\hline & $p$ & 0.030 & 0.027 & 0.037 & 0.447 & 0.884 & 0.991 \\
\hline \multicolumn{8}{|l|}{ GCC thickness $(\mu \mathrm{m})$} \\
\hline \multirow{2}{*}{ Superior } & $r_{5}$ & -0.35 & -0.31 & 0.06 & 0.19 & 0.11 & -0.12 \\
\hline & $\mathrm{p}$ & 0.014 & 0.029 & 0.671 & 0.184 & 0.474 & 0.417 \\
\hline \multirow[b]{2}{*}{ Total } & $r_{5}$ & -0.31 & -0.27 & 0.07 & 0.20 & 0.10 & -0.07 \\
\hline & $p$ & 0.032 & 0.050 & 0.647 & 0.166 & 0.492 & 0.629 \\
\hline \multicolumn{8}{|l|}{ Macular thickness $(\mu \mathrm{m})$} \\
\hline \multirow{2}{*}{ Parafovea temporal } & $r_{s}$ & 0.03 & 0.04 & -0.27 & 0.01 & -0.25 & -0.01 \\
\hline & $\mathrm{p}$ & 0.827 & 0.790 & 0.041 & 0.936 & 0.059 & 0.970 \\
\hline \multirow{2}{*}{ Parafovea nasal } & $r_{5}$ & 0.02 & 0.03 & -0.17 & 0.01 & -0.27 & -0.04 \\
\hline & $\mathrm{p}$ & 0.883 & 0.797 & 0.059 & 0.998 & 0.041 & 0.795 \\
\hline \multirow{2}{*}{ Fovea } & $r_{5}$ & -0.13 & -0.10 & -0.43 & 0.12 & -0.30 & -0.10 \\
\hline \multirow{2}{*}{\multicolumn{8}{|c|}{$\begin{array}{l}\text { OCTA parameter } \\
\text { Superficial vessel density, \% }\end{array}$}} \\
\hline & & & & & & & \\
\hline \multirow{2}{*}{ Fovea } & $r_{5}$ & -0.11 & -0.09 & -0.23 & 0.14 & -0.15 & -0.15 \\
\hline & $\frac{5}{p}$ & 0.324 & 0.404 & 0.040 & 0.220 & 0.189 & 0.167 \\
\hline \multicolumn{8}{|l|}{ Deep vessel density, \% } \\
\hline \multirow{2}{*}{ Whole-en-face } & $r_{5}$ & -0.02 & -0.01 & -0.07 & -0.01 & -0.03 & .22 \\
\hline & $\mathrm{p}$ & 0.881 & 0.934 & 0.540 & 0.953 & 0.762 & 0.042 \\
\hline \multirow{2}{*}{ Fovea } & $r_{s}$ & -0.14 & -0.13 & -0.20 & 0.21 & -0.05 & -0.06 \\
\hline & $p$ & 0.202 & 0.244 & 0.065 & 0.050 & 0.658 & 0.615 \\
\hline \multirow{2}{*}{ Foveal avascular zone, $\mathrm{mm}^{2}$} & $r_{5}$ & 0.01 & 0.00 & .26 & -0.11 & 0.11 & -0.05 \\
\hline & $p$ & 0.938 & 0.969 & 0.022 & 0.326 & 0.320 & 0.644 \\
\hline
\end{tabular}

In this study, although the thickness of GCC was similar among the PCOS patients and healthy controls, increased parafoveal thickness in women with PCOS can be attributed to reactive astrogliosis due to elevated insulin levels and hyperglycemia. The number and size of astrocytes and cytoskeletal components have been shown to increase in such cases. ${ }^{16}$

It has been well documented that steroid hormones increase the ocular blood flow. ${ }^{4}$ In a study, Atalay et al. examined the impacts of hormone replacement therapy on ocular blood flow and found that the central retinal artery pulsatility index values significantly decreased at three and six months. ${ }^{17}$ In another study, Toker et al. evaluated the effect of sex hormones on ocular hemodynamics on pre- and post-menopausal women; and observed a positive, significant correlation between elevated testosterone levels and resistive index of the central retinal artery. ${ }^{18}$ Some authors have also suggested that vascular tone is shifted from vasodilatation toward vasoconstriction due to hyperandrogenism in the early phase of PCOS. ${ }^{19}$ Chekir et al. evaluated uterine perfusion and its relationship with clinical and biochemical parameters in PCOS patients and found significantly higher uterine arterial pulsatility index in the patient group compared to healthy controls. ${ }^{20}$ In another study, Sahu et al. found an inverse correlation between LH, TT, serum fasting insulin levels and ovarian stromal arterial pulsatility index in patients with PCOS. ${ }^{21}$ Oner et al. reported that ocular pulse amplitude decreased in obese patients compared to healthy controls, and also showed a negative correlation with IR and could affect ocular blood flow. ${ }^{22}$ In a study evaluating whether the presence of PCOS altered ocular blood flow parameters using color Doppler images, Ornek et al. found that ocular blood flow velocity increased in women with PCOS and vascular resistance decreased only in the ophthalmic artery. ${ }^{6}$ Different from previous studies, the present authors evaluated capillarys of the macula and optic disc through non-invasive OCTA and found no significant difference between the PCOS patient and healthy controls. Of note, some authors have proposed that OCTA is useful in identifying early vascular alterations in patients with diabetes. ${ }^{23}$ In a study evaluating vessel density in children with type 1 diabetes having no signs of diabetic retinopathy by OCTA, Golçbiewska et al. showed no statistically significant difference in the SCP and DCP among diabetic and healthy children. ${ }^{24}$ In a similar study using OCTA images, however, Mameli et al. reported significantly lower capillary densities in SCP and DCP with diabetic patients compared to the control group. ${ }^{25}$ There was no statistically significant difference in retinal capillaries between PCOS and healthy women in this study when compared with the control group, consistent with the study of Golcbiewska et al. ${ }^{24}$ This can be attributed to the fact that, despite increased IR, this study cohort included younger PCOS patients with unknown clinical diabetes, obesity, or dyslipidemia without morphological alterations in the retinal capillary network yielding numerical vessel density data. Furthermore, it was observed a significant correlation between the lipid profile and FAZ, macular capillary network. This finding suggests that PCOS-related endocrine and metabolic alterations may induce OCTA abnormalities in the long-term.

\section{CONCLUSION}

Although no microvascular macular abnormalities were seen in PCOS patients, the parafoveal thickness significantly increased in all quadrant except the nasal quadrant. In addition, morphovascular parameters of the optic disc region were similar between the PCOS patients and healthy controls.

\section{ETHICAL APPROVAL:}

The study protocol was approved by the local Ethics Committee (No. 2019 /01-39; dated: 02/01/2019). 


\section{PATIENTS' CONSENT:}

Written informed consents were obtained from all participants before any examination.

\section{CONFLICT OF INTEREST:}

The authors declared no conflict of interest.

\section{AUTHORS' CONTRIBUTION:}

NPY, GO, AT: Conception and design analysis.

LO, GAA: Acquisition, analysis, interpretation of data.

\section{REFERENCES}

1. Azhar A, Abid F, Rehman R. Polycystic Ovary Syndrome, Subfertility and Vitamin D Deficiency. J Coll Physicians Surg Pak 2020; 30(5):545-6. doi: 10.29271/jcpsp.2020.05.545.

2. Rotterdam ESHRE/ASRM-Sponsored PCOS consensus workshop group. Revised 2003 consensus on diagnostic criteria and long-term health risks related to polycystic ovary syndrome (PCOS). Hum Reprod 2004; 19(1):41-7. doi: 10.1093/humrep/deh098.

3. Nuzzi R, Scalabrin S, Becco A, Panzica G. Gonadal Hormones and Retinal Disorders: A Review. Front Endocrinol (Lausanne) 2016; 9:66. doi: 10.3389/fendo.2018.00066.

4. Açmaz G, Ataş M, Gülhan A, Açmaz B, Ataş F, Aksoy H. Evaluation of the macula, retinal nerve fiber layer, and choroid thickness in women with polycystic ovary syndrome using spectral-domain optical coherence tomography. Reprod Sci 2014; 21(8):1044-9. doi: 10.1177/1933719114522523.

5. Demir M, Guven D, Koc A, Ozdemir S, Can E. Retinal nerve fiber layer thickness in women with polycystic ovary syndrome. J Ophthalmol 2013; 2013:752186. doi: 10.1155/ 2013/752186.

6. Örnek N, Inal M, Tulmaç ÖB, Özcan-Dağ Z, Örnek K. Ocular blood flow in polycystic ovary syndrome. J Obstet Gynaecol Res 2015; 41(7):1080-6. doi: 10.1111/jog.12673.

7. Spaide RF, Fujimoto JG, Waheed NK, Sadda SR, Staurenghi G. Optical coherence tomography angiography. Prog Retin Eye Res 2018;64: 1-55. doi: 10.1016/j.preteyeres.2017.11. 003.

8. De Souza-Júnior JE, de Amorim Garcia CA, Soares EMM. Polycystic ovary syndrome: aggressive or protective factor for the retina? evaluation of macular thickness and retinal nerve fiber layers using high-definition optical coherence tomography. J Ophthalmol 2015; 2015:193078. doi: 10. 1155/2015/193078.

9. Bialek M, Zaremba P, Borowicz KK, Czuczwar SJ. Neuroprotective role of testosterone in the nervous system. Pol J Pharmacol 2004; 56(5):509-18.

10. Ansari SA, Emerald BS. The role of Insulin resistance and protein 0 -GICNAcylation in neurodegeneration. Front Neurosci 2019; 13:473. doi: 10.3389/fnins.2019.00473. eCollection 2019.

11. Nakamura M, Barber AJ, Antonetti DA, LaNoue KF, Robinson KA, Buse MG, et al. Excessive hexosamines block the neuroprotective effect of insulin and induce apoptosis in retinal neurons. J Biol Chem 2001; 276:43748-55. doi: 10.1074/ jbc.M108594200.

12. Pierro L, Iuliano L, Cicinelli MV, Casalino G, Bandello F.
Retinal neurovascular changes appear earlier in type 2 diabetic patients. Eur J Ophthalmol 2017; 27(3):346-51. doi: 10.5301/ejo.5000887.

13. Srinivasan S, Pritchard N, Sampson GP, Edwards K, Vagenas D, Russell AW, et al. Retinal thickness profile of individuals with diabetes. Ophthalmic Physiol 2016; Opt 36(2):158-66. doi: 10.1111/opo.12263.

14. Toft-Kehler AK, Skytt DM, Kolko M. A Perspective on the Müller Cell-Neuron Metabolic Partnership in the Inner Retina. Mol Neurobiol 2018; 55(6):5353-61. doi: 10.1007/ s12035-017-0760-7.

15. Rübsam A, Parikh S, Fort PE. Role of Inflammation in Diabetic Retinopathy. Int J Mol Sci 2018; 19(4):942. doi: 10. 3390/ijms19040942.

16. De Hoz R, Rojas B, Ramírez Al, Salazar JJ, Gallego BI, Triviño $A$, et al. Retinal Macroglial Responses in Health and Disease. Biomed Res Int 2016; 2016:2954721. doi: 10.1155/ 2016/2954721.

17. Atalay E, Karaali K, Akar M, Seker Ari E, Simsek M, Atalay S, et al. Early impact of hormone replacement therapy on vascular hemodynamics detected via ocular colour Doppler analysis. Maturitas 2005;50(4): 282-8. doi: 10.1016/j.maturitas.2004.06.023.

18. Toker E, Yenice O, Akpinar I, Aribal E, Kazokoglu H. The influence of sex hormones on ocular blood flow in women. Acta Ophthalmol Scand 2003;81(6):617-24. doi: 10.1111/j.1395-3907.2003.00160.x.

19. Masszi G, Horvath EM, Tarszabo R, Benko R, Novak A, Buday $A$, et al. Reduced estradiol induced vasodilation and poly(ADP-ribose) polymerase (PARP) activity in the aortas of rats with experimental polycystic ovary syndrome (PCOS). PLOS One 2013; 8(3):e55589. doi: 10.1371/journal.pone 0055 589.

20. Chekir C, Nakatsuka M, Kamada Y, Noguchi S, Sasaki A, Hiramatsu $Y$. Impaired uterine perfusion associated with metabolic disorders in women with polycystic ovary syndrome. Acta Obstet Gynecol Scand 2005; 84(2):189-95. doi: 10.1111/j.0001-6349.2005.00678.x.

21. Sahu A, Tripathy P, Mohanty J, Nagy A. Doppler analysis of ovarian stromal blood flow changes after treatment with metformin versus ethinyl estradiol-cyproterone acetate in women with polycystic ovarian syndrome: A randomized controlled trial. J Gynecol Obstet Hum Reprod 2019; 48(5):335-9. doi: 10.1016/j.jogoh.2018.10.006.

22. Öner Ri, Karadağ AS. Evaluation of choroidal perfusion changes in obese patients: ocular effects of insulin resistance. Arq Bras Oftalmol 2018; 81(6):461-5. doi: 10.5935/ 0004-2749.20180088.

23. Cao D, Yang D, Huang Z, Zeng Y, Wang J, Hu Y, et al. Optical coherence tomography angiography discerns preclinical diabetic retinopathy in eyes of patients with type 2 diabetes without clinical diabetic retinopathy. Acta Diabetol 2018; 55(5):469-77. doi: 10.1007/s00592-018-1115-1.

24. Goøębiewska J, Olechowski A, Wysocka-Mincewicz M, Odrobina D, Baszyńska-Wilk M, Groszek A, et al. Optical coherence tomography angiography vessel density in children with type 1 diabetes. PLoS One 2017; 12(10): e0186479. doi: 10.1371/journal.pone.0186479. eCollection 2017. 
25. Mameli C, Invernizzi A, Bolchini A, Bedogni G, Giani E, Macedoni $M$, et al. Analysis of retinal perfusion in children, adolescents, and young adults with type 1 diabetes using optical coherence tomography angiography. J Diabetes Res 2019;

2019:5410672. doi: 10.1155/2019/5410672. eCollection 2019.

$\bullet \bullet \bullet \bullet \bullet \bullet \bullet \bullet \bullet$ 